\title{
Long-term follow-up of a randomized AAV2-CAD gene therapy trial for Parkinson's disease
}

Martin Niethammer, ${ }^{1}$ Chris C. Tang, ${ }^{1}$ Peter A. LeWitt, ${ }^{2}$ Ali R. Rezai, ${ }^{3}$ Maureen A. Leehey, ${ }^{4}$ Steven C. Ojemann, ${ }^{4}$ Alice W. Flaherty, ${ }^{5}$ Emad N. Eskandar, ${ }^{5,6}$ Sandra K. Kostyk, ${ }^{3}$ Atom Sarkar, ${ }^{7}$ Mustafa S. Siddiqui, ${ }^{8}$ Stephen B. Tatter, ${ }^{8}$ Jason M. Schwalb, ${ }^{9}$ Kathleen L. Poston, ${ }^{10,11}$ Jaimie M. Henderson, ${ }^{11}$ Roger M. Kurlan, ${ }^{12}$ Irene H. Richard, ${ }^{13}$ Christine V. Sapan, ${ }^{14}$ David Eidelberg, Matthew J. During, ${ }^{3,15}$ Michael G. Kaplitt, ${ }^{16}$ and Andrew Feigin ${ }^{1}$

'Center for Neurosciences, The Feinstein Institute for Medical Research, Manhasset, New York, USA. 2Parkinson's Disease and Movement Disorders Program, Henry Ford Hospital, West Bloomfield, Michigan, USA; Department of Neurology, Wayne State University School of Medicine, Detroit, Michigan, USA. ${ }^{3}$ Department of Neurological Surgery, The Ohio State University College of Medicine, Columbus, Ohio, USA. ${ }^{4}$ Department of Neurology, University of Colorado School of Medicine, Aurora, Colorado, USA. ${ }^{5}$ Department of Neurology and ${ }^{6}$ Department of Neurosurgery, Massachusetts Ceneral Hospital, Boston, Massachusetts, USA. 'Department of Neurosurgery, Geisinger Health System, Danville, Pennsylvania, USA. ${ }^{8}$ Department of Neurology, Wake Forest School of Medicine, Winston-Salem, North Carolina, USA. ${ }^{9}$ Movement Disorder \& Comprehensive Epilepsy Centers, Henry Ford Medical Group, West Bloomfield, Michigan, USA. ${ }^{10}$ Department of Neurology and Neurological Sciences and "Department of Neurosurgery, Stanford University School of Medicine, Stanford, California, USA. ${ }^{12}$ Neurology, The Center for Neurological and Neurodevelopmental Health, Voorhees, New Jersey, USA. ${ }^{13}$ Department of Neurology and Department of Psychiatry, University of Rochester School of Medicine and Dentistry, Rochester, New York, USA. ${ }^{14}$ Asana Medical Inc., Miami Lakes, Florida, USA. ${ }^{15}$ Department of Molecular Virology, Immunology, and Medical Genetics, The Ohio State University College of Medicine, Columbus, Ohio, USA. ${ }^{16}$ Department of Neurological Surgery, Weill Cornell Medical College, New York, New York, USA.

Role of funding source: The sponsor did not play a role in study design, collection, analysis and interpretation of data, writing of the report, or in the decision to submit the paper for publication.

Conflict of interest: C.C. Tang, P.A. Le Witt, M.A. Leehey, S.C. Ojemann, A.W. Flaherty, S.K. Kostyk, M.S. Siddiqui, S.B. Tatter, J.M. Schwalb, K.L. Poston, I.H. Richard, M.J. During, and M.G. Kaplitt have received funding from funding from Neurologix Inc. M.J. During and M.G. Kaplitt are coinventors on the patent re: Glutamic acid decarboxylase (GAD) based delivery systems (United States Patent No. 7,695,959 B2). D. Eidelberg is a coinventor on the patents re: Markers for use in screening patients for nervous system dysfunction and a method and apparatus for using same (United States Patent No. 5,632,276 and No. $5,873,823)$. Additional COI information is reported in the supplemental materials.

Submitted: August 12, 2016 Accepted: February 23, 2017 Published: April 6, 2017

\section{Reference information:}

JCI Insight. 2017;2(7):e90133. https:// doi.org/10.1172/jci.insight.90133.
BACKGROUND. We report the 12-month clinical and imaging data on the effects of bilateral delivery of the glutamic acid decarboxylase gene into the subthalamic nuclei (STN) of advanced Parkinson's disease (PD) patients.

METHODS. 45 PD patients were enrolled in a 6-month double-blind randomized trial of bilateral AAV2-CAD delivery into the STN compared with sham surgery and were followed for 12 months in open-label fashion. Subjects were assessed with clinical outcome measures and ${ }^{18}$ F-fluorodeoxyglucose (FDG) PET imaging.

RESULTS. Improvements under the blind in Unified Parkinson's Disease Rating Scale (UPDRS) motor scores in the AAV2-CAD group compared with the sham group continued at 12 months [time effect: $F(4,138)=11.55, P<0.001$; group effect: $F(1,35)=5.45, P<0.03$; repeatedmeasures ANOVA (RMANOVA)]. Daily duration of levodopa-induced dyskinesias significantly declined at 12 months in the AAV2-CAD group ( $P=0.03$; post-hoc Bonferroni test), while the sham group was unchanged. Analysis of all FDC PET images over 12 months revealed significant metabolic declines ( $P<0.001$; statistical parametric mapping RMANOVA) in the thalamus, striatum, and prefrontal, anterior cingulate, and orbitofrontal cortices in the AAV2-CAD group compared with the sham group. Across all time points, changes in regional metabolism differed for the two groups in all areas, with significant declines only in the AAV2-CAD group ( $P<0.005$; post-hoc Bonferroni tests). Furthermore, baseline metabolism in the prefrontal cortex (PFC) correlated with changes in motor UPDRS scores; the higher the baseline PFC metabolism, the better the clinical outcome.

CONCLUSION. These findings show that clinical benefits after gene therapy with STN AAV2$C A D$ in PD patients persist at 12 months. 
TRIAL REGISTRATION. ClinicalTrials.gov NCT00643890.

FUNDING. Neurologix Inc.

\section{Introduction}

The motor features of Parkinson's disease (PD) primarily result from the loss of nigrostriatal dopaminergic neurons, which are part of a distributed basal ganglia network. As a consequence of their loss, the diminished GABAergic input from the pallidum leaves the subthalamic nucleus (STN) disinhibited (1). Therapies aimed at modulating STN activity can improve the motor features of PD.

Dopaminergic replacement therapies are effective for many motor impairments of PD, but over time, a subset of patients develop increasingly severe fluctuations in levodopa benefit and suboptimal overall control of parkinsonism (2). In these circumstances, deep brain stimulation (DBS) of the STN can be quite effective, although its use can be associated with adverse events and requires indwelling hardware (3). Hence, improved outcomes are desirable for therapeutic approaches targeting this brain pathway. Gene therapy has shown promise in human therapeutic applications, with increasing evidence for its safety and efficacy (4-6). We conducted a randomized, double-blind, sham surgery-controlled clinical trial of a gene therapy utilizing a combination of genes for glutamic acid decarboxylase (GAD65 and GAD67), the rate-limiting enzyme for the production of GABA, delivered via an adeno-associated-2 viral vector (AAV2) into the bilateral STN (AAV2-GAD) (4). The therapeutic goal was a transformation of glutamatergic to GABAergic inhibitory modulation of STN neuronal activity, thereby improving the motor features of PD. Indeed, this trial produced promising clinical results, with improvement in both the primary outcome measure (change in motor Unified Parkinson's Disease Rating Scale [UPDRS] scores over the 6-month blinded study period) and several secondary outcome measures (4). Subjects in this 6-month randomized, double-blind, sham-controlled trial were followed in an open-label fashion through 12 months, with both clinical and imaging ( ${ }^{18} \mathrm{~F}$-fluorodeoxyglucose [FDG] PET) outcome measures. We now report the 12-month clinical and imaging data from this trial.

\section{Results}

Clinical. There was no difference ( $P=0.12$; Student's $t$ test) in the UPDRS motor scores between the AAV2$G A D(34.8 \pm 6.6[$ mean $\pm \mathrm{SD}])$ and sham $(38.9 \pm 8.8)$ groups at baseline (4). After receiving gene therapy, the improved UPDRS motor scores observed during the first 6 months in the AAV2-GAD treatment group relative to the sham group (see Figure 2 in ref. 4) were maintained at 12 months (Figure 1A), as shown by a significant decline over time [time effect: $F(4,138)=11.55, P<0.001$ ] and a significant difference between groups across all 4 postoperative time points [group effect: $F(1,35)=5.45, P<0.03 ; 2 \times 5$ repeated-measures ANOVA (RMANOVA)]. Nonetheless, the trajectories of decreases in the UPDRS motor scores were found to be parallel for the two groups [group by time interaction: $F(4,138)=0.72, P=0.58$ ] . In both groups, post-hoc comparisons further revealed that, relative to baseline, declines in the UPDRS motor scores were significant at 1 month $(P<0.05)$ and persisted until 12 months $(P<0.01)$.

There was no difference at baseline in the answers to the UPDRS part 4 questions related to duration of levodopa-induced dyskinesias (LID) (item 32; $P=0.09$; Student's $t$ test) or duration of OFF time (item 39; $P=0.07$ ). Changes in duration of LID differed over time between the two groups (Figure 1B; interaction effect: $P<0.02 ; 2 \times 5$ RMANOVA), with a significant decline at 12 months relative to baseline in the AAV2-GAD group ( $P=0.03$; post-hoc Bonferroni test) and no changes in the sham group. Duration of OFF time was observed to be relatively shorter in the AAV2-GAD group compared with the sham group at all time points, albeit nonsignificantly (data not shown; interaction effect: $P=$ 0.12; group effect: $P=0.47 ; 2 \times 5$ RMANOVA).

Finally, we analyzed responder rates based on a number of clinical endpoints. As in the 6-month data (4), a cutpoint of improvement by 9.0 points in the UPDRS motor scores (a $25 \%$ improvement from the average baseline score) identified a significantly greater $\left(\chi^{2}=5.64, P<0.02\right)$ responder rate in the AAV2-GAD group (10 of $16,62.5 \%$ ) than in the sham group (5 of $21,23.8 \%$ ) (Figure $1 C$ ). Seven of the eight subjects in the AAV2-GAD group that were classified as responders at 6 months, remained responders at 12 months (in contrast with 1 of 3 subjects in the sham group). 
A

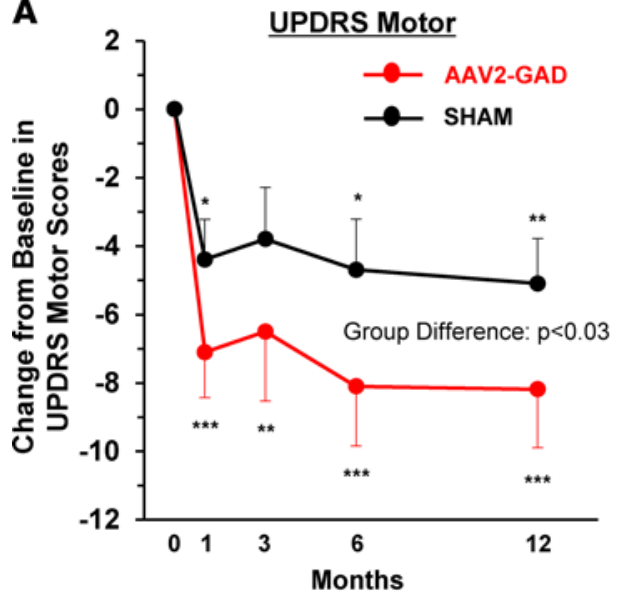

C

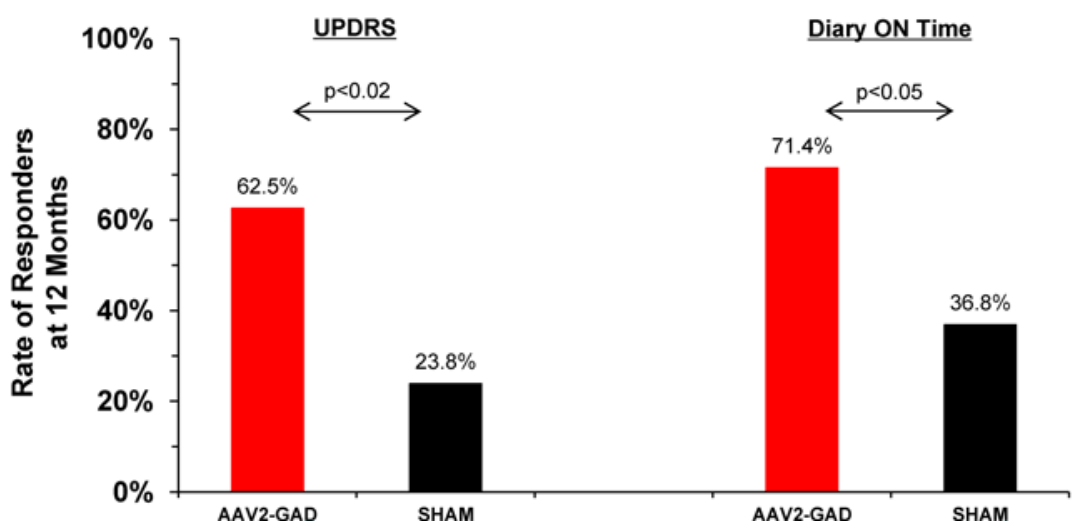

Figure 1. Clinical improvements after gene therapy. (A) Changes in mean OFF-state Unified Parkinson's Disease Rating Scale (UPDRS) motor scores at baseline and 1, 3, 6, and 12 months in the AAV2-CAD (red, $n=$ 16) and sham (black, $n=21$ ) groups. After surgery, the patients in both groups showed decreased scores over time (time effect: $P<0.001$ by 2-way RMANOVA), with greater improvements in the AAV2-GAD treatment group over all follow-up time points (group effect: $P<0.03 ; 2 \times 5$ RMANOVA; ${ }^{*} P<0.05,{ }^{* *} P<0.01$, ${ }^{* * *} P<$ 0.001 , post-hoc Bonferroni tests relative to baseline). (B) Changes in the duration of levodopa-induced dyskinesias (LID) (item 32 of the UPDRS) in the AAV2-CAD (red, $n=16$ ) and sham (black, $n=21$ ) groups over the course of the study. There was a significant difference in duration of LID over time between the two groups (interaction effect: $P<0.02,2 \times 5$ RMANOVA). (C) Rate of responders and nonresponders at 12 months. A cutpoint of improvement of 9.0 points identified a significantly greater $\left(\chi^{2}=5.64, P<0.02\right)$ responder rate in the AAV2-CAD group ( 10 of $16,62.5 \%$ ) than in the sham group ( 5 of $21,23.8 \%$ ) at both time points. $A$ cutpoint improvement of 1 hour for diary-based $O N$ time revealed a significant difference between the two groups $\left(\chi^{2}=3.86, P<0.05\right)$, with a larger percentage in the AAV2-GAD group ( 10 of $14,71.4 \%$ ) categorized as responders than in the sham group (7 of 19, 36.8\%). Two subjects each in the AAV2-CAD and sham groups were missing 12-month diary data and were omitted from this analysis.

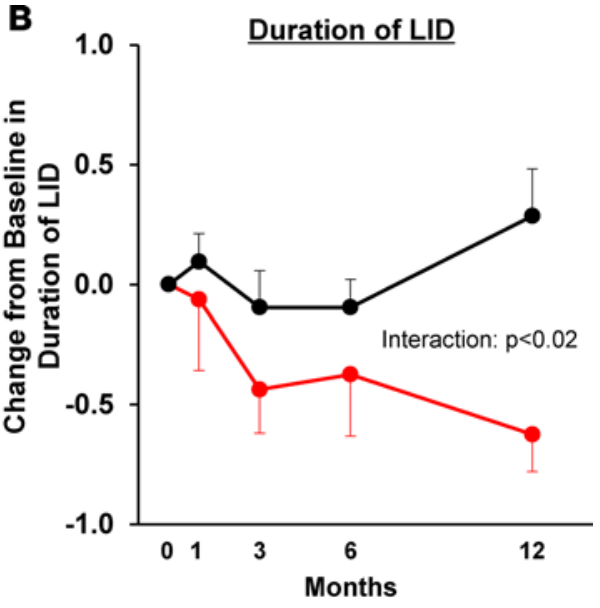

There was no significant difference between groups over time in diary measures of ON time $(P=0.97)$, OFF time $(P=0.83)$, and ON time with $\operatorname{LID}(P=$ $0.90)$ when assessed with RMANOVA. Nevertheless, using an improvement of 1 hour as a cutpoint for diary-based ON times revealed a significant difference between the two groups. A larger percentage of subjects in the AAV2-GAD group (10 of $14,71.4 \%$ ) was categorized as responders than in the sham group (7 of $19,36.8 \% ; \chi^{2}=3.86, P<$ 0.05) (Figure 1C). Moreover, more AAV2-GAD subjects were classified as both UPDRS and ON time responders than sham subjects (5 of 14 [35.7\%] in the AAV2-GAD group versus 1 of 19 [5.3\%] in the sham group). There was no significant difference in diary OFF time, using a 1-hour improvement as a cutpoint $\left(\chi^{2}=2.43, P=0.12\right)$.

Imaging. In a separately published analysis, we found no significant differences in metabolic profiles among the different imaging sites, regardless of the scanner used (7). Voxel-based statistical parametric mapping (SPM) analysis of all FDG PET images over the course of the study revealed significant metabolic declines $(P<0.001$, uncorrected; SPM $2 \times 3$ RMANOVA) in the thalamus, striatum, prefrontal cortex (BA $8 / 9 / 10$ ), anterior cingulate cortex (BA $23 / 24$ ), and orbitofrontal cortex (BA $47)$ in the AAV2-GAD group compared with the sham group (Table 1 and Figure $2 \mathrm{~A}$ ). In each of these areas, there was no difference in regional metabolism between the two groups at baseline ( $P>0.07$; Student's $t$ tests). Moreover, in all of these areas except the striatum,

baseline metabolic activity in both groups did not differ from the metabolism measured in the same areas in a group of age-matched healthy control subjects ( $P>0.24$; Student's $t$ tests). Striatal metabolism at baseline was significantly lower in the sham group compared to the healthy control group $(P<0.02$; Student's $t$ test).

Across all time points, changes in regional metabolism were different for the two groups in all areas (Figure 2B; interaction effect: $P<0.005 ; 2 \times 3$ RMANOVA), with significant declines over time in the AAV2-GAD group ( $P<0.005$; post-hoc Bonferroni tests) and no changes in the sham group.

Further analysis showed that there was a significant inverse correlation between baseline metabolism in the prefrontal cortex and the changes in UPDRS motor scores at 6 months (Figure 3A: $\mathrm{r}=$ $-0.51, P<0.05$ ) and 12 months (Figure 3B: $\mathrm{r}=-0.71, P<0.003$ ) in the AAV2-GAD group but not in the sham group at either follow-up time point (Figure 3, C and $D: P \geq 0.12$ ). Such correlations were nonsignificant in both groups for all other regions at both $6(P>0.07)$ and 12 months $(P>0.19)$. No other baseline regional metabolism correlated with clinical outcome. 
Table 1. Brain regions with significant differences in changes in metabolism from baseline to 6 and 12 months between the AAV2-GAD and sham groups

\begin{tabular}{|c|c|c|c|c|c|}
\hline \multirow{2}{*}{ Brain region } & \multicolumn{3}{|c|}{ MNI coordinates } & \multirow{2}{*}{$Z_{\text {max }}{ }^{A}$} & \multirow{2}{*}{ Cluster extent } \\
\hline & $x$ & $y$ & $z$ & & \\
\hline \multicolumn{6}{|c|}{$\Delta$ Group AAV2-GAD $<\Delta$ Group Sham } \\
\hline Thalamus & -2 & -22 & 0 & 3.86 & 515 \\
\hline \multicolumn{6}{|c|}{ Caudate/putamen } \\
\hline Left & -14 & 0 & 14 & 6.06 & 621 \\
\hline Right & 18 & -4 & 16 & 5.76 & 1,071 \\
\hline BA 10 & 28 & 56 & 8 & 3.69 & 128 \\
\hline \multicolumn{6}{|c|}{ Anterior cingulate/PFC } \\
\hline BA $24 / 9$ & 6 & 14 & 30 & 5.55 & 4,324 \\
\hline BA 23 & -6 & -24 & 36 & 4.29 & 163 \\
\hline \multicolumn{6}{|c|}{ Orbitofrontal cortex } \\
\hline
\end{tabular}

${ }^{A} P<0.001$, uncorrected, SPM RMANOVA. $\triangle$, changes in regional metabolism from baseline. MNI, Montreal Neurological Institute; PFC, prefrontal cortex.

\section{Discussion}

As we reported in an earlier publication (4), PD subjects in this study receiving AAV2-GAD gene therapy improved compared with the sham treatment group, and these benefits persisted for 12 months following the surgical procedure, with significant improvements in UPDRS scores. Other clinical outcome measures, including daily duration of LID and percentage of subjects achieving a clinically meaningful improvement in UPDRS score or ON time (responder analysis), also continued to demonstrate benefit from the AAV2$G A D$ treatment at 12 months. In addition, while $\mathrm{ON}$ time was not used as a study entry criterion, we note that 5 of the AAV2-GAD-treated nonresponders, according to the UPDRS criteria, showed an increase in ON time by over 2 hours, suggesting that different clinical measures may capture different aspects of clinically meaningful improvements in PD subjects. Although this phase II study was not primarily powered for efficacy, several of the findings over 1 year of follow-up, including the primary outcome measure (change in UPDRS motor score), improved ON time, and responder analyses, provide strong support for the potential efficacy of STN AAV2-GAD in PD.

We note that the mean level of improvement in the STN AAV2-GAD group was less than that seen with some current therapies for advanced PD such as DBS (8), but, given the lack of active comparators in this study and the relatively small sample size, the relative efficacy of STN AAV2-GAD will need to be further assessed in future studies powered for efficacy. For example, the mean decrease in UPDRS motor score and its $95 \%$ confidence interval for the AAV2-GAD group at 12 months was $-8.2 \pm 3.3$ (or $23.6 \% \pm$ $9.5 \%$ of the baseline mean), corresponding to a range of between -11.5 and -4.9 (or $33.0 \%$ to $14.1 \%$ of the baseline); this range encompasses levels of improvement seen with other therapies. Interestingly, the sham group also exhibited a sustained benefit in UPDRS scores, albeit less than that of the AAV2-GAD group. We have recently identified a metabolic brain network relating to a sham response; moreover, expression of this network at baseline appears to indicate subjects that are "sham susceptible" (9).

FDG PET imaging was utilized in this study to both strengthen confidence in the accuracy of the diagnosis of idiopathic $\mathrm{PD}$ prior to enrolling subjects and to provide further insights into the mechanisms underlying the effects of placing AAV2-GAD in the STN. The first goal, enhancing the certainty of a correct diagnosis in this small phase II trial, was critical, since the therapeutic intervention was not expected to be of benefit to individuals with atypical parkinsonism. Indeed, 11 subjects were excluded from the study on the basis of these scan results, including 4 subjects demonstrated to have unequivocal multiple system atrophy (MSA) by FDG PET imaging. Prior clinical trials that utilized dopaminergic imaging $\left({ }^{18} \mathrm{~F}\right.$-fluorodopa or dopamine transporter system imaging) have reported evidence that $10 \%-15 \%$ of subjects that were categorized by clinical criteria as having idiopathic PD lack findings of a dopaminergic deficit (10-12). However, these studies may underestimate the misdiagnosis rate, since dopaminergic imaging does not distinguish 
A

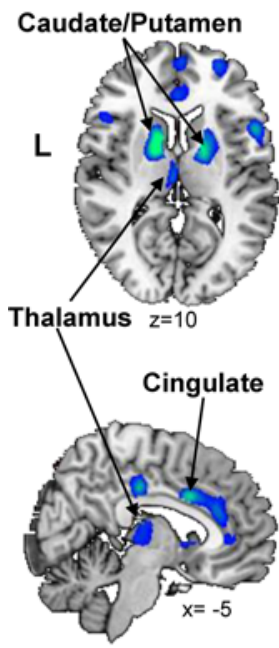

B

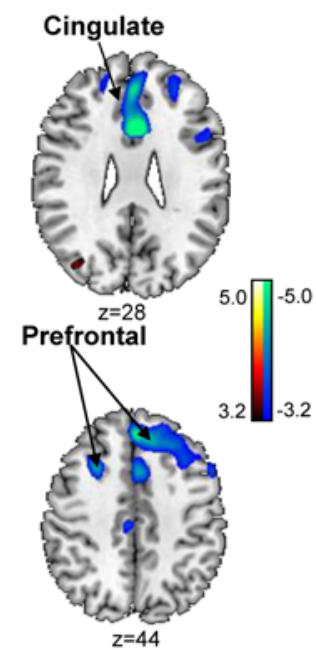

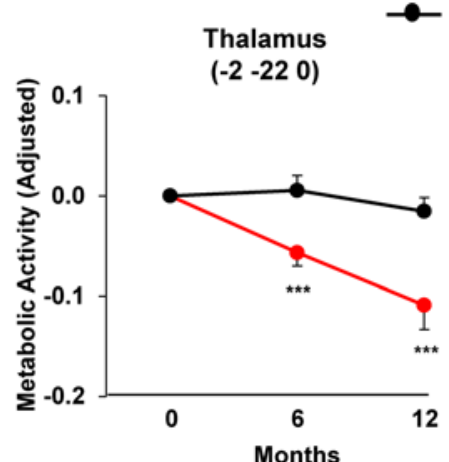
(28 56 8)

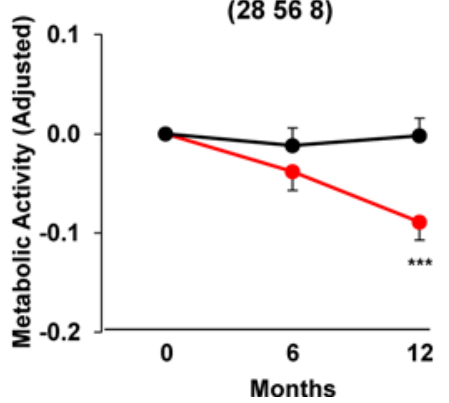

AAV2-GAD

SHAM

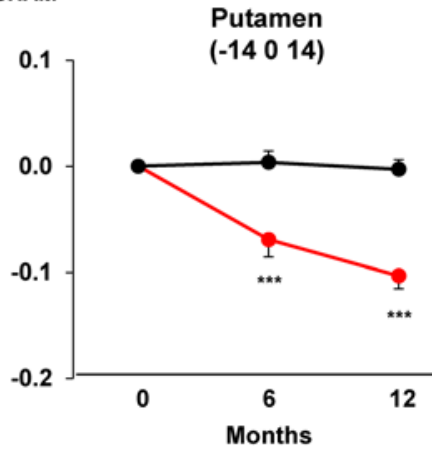

Anterior Cingulate

(6 14 30)

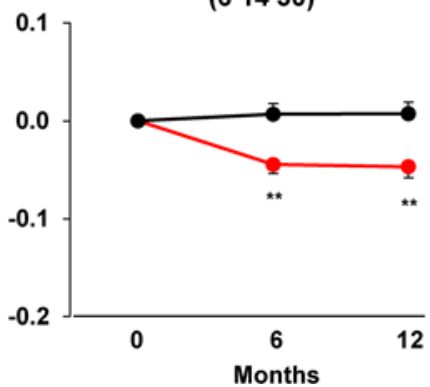

Figure 2. Changes in regional metabolism after gene therapy. (A) Representative slices displaying significant changes in glucose metabolism in the thalamus, caudate/putamen, prefrontal cortex (BA 8/9/10), and anterior cingulate cortex (BA 23/24) in the AAV2-CAD group ( $n=16$ ) compared with the sham group ( $n=21)$ after gene therapy $(P<0.001$, uncorrected; SPM RMANOVA). (Increased/decreased metabolism is indicated by red/blue.) (B) Mean metabolic activity in these regions was plotted for the AAV2-CAD (red, $n=16$ ) and sham (black, $n=21$ ) groups. In all of these areas, changes in regional metabolism were different for the two groups (interaction effect: $P<0.005 ; 2 \times 3$ RMANOVA), with significant declines over time only in the AAV2-GAD group ( $P<$ $0.005 ;{ }^{* *} P<0.01 ;{ }^{* *} P<0.001$, post-hoc Bonferroni tests relative to baseline).

idiopathic PD from atypical forms of parkinsonism. Even in this study, which enrolled advanced levodopa-responsive subjects meeting strict clinical criteria, we found that approximately $20 \%$ of subjects screened probably did not have PD based on imaging findings. Since the misdiagnosis rate might be even higher in mildly affected parkinsonian subjects, these data suggest that future small early phase trials would be well advised to consider utilizing an objective imaging measure such as FDG PET in the screening process.

The changes in FDG PET imaging in response to AAV2-GAD may provide some insights into the mechanisms underlying the therapy. Notably, in the AAV2-GAD treatment group, there were dramatic declines in brain metabolism (in thalamus, striatum, anterior cingulate, and prefrontal cortex) not seen in the sham group; these changes were far removed from the surgical site and therefore likely represent significant functional changes related to alteration in STN activity. The decline in thalamic metabolism is of particular note, as this was the primary observation in the treated hemispheres of patients in our prior open-label trial of AAV2-GAD treatment (13), and declines in thalamic metabolism would be an expected downstream effect of STN suppression. Indeed, several studies have demonstrated relative increases in thalamic and putaminal metabolism in PD and suppression of this activity with effective symptomatic therapies, such as levodopa and STN DBS $(14,15)$. The consistency of the effect over time in the thalami of patients in our phase I study and in the current study, along with the absence of effect in the untreated hemispheres of phase I patients and control patients here, strongly indicates that decline in thalamic metabolism is a genuine consequence of AAV2-GAD gene therapy surgery.

We also found metabolic declines in anterior cingulate and prefrontal cortices in the AAV2-GAD group. According to a widely accepted model of basal ganglia circuitry, STN modulation (whether by DBS or lesioning) would be expected to facilitate output of signal projections to cortical structures (16, 17). Nonetheless, decreased activity in the anterior cingulate cortex has been observed in both the resting state and during task activation following STN DBS $(16,18)$, perhaps through the disruption of the 

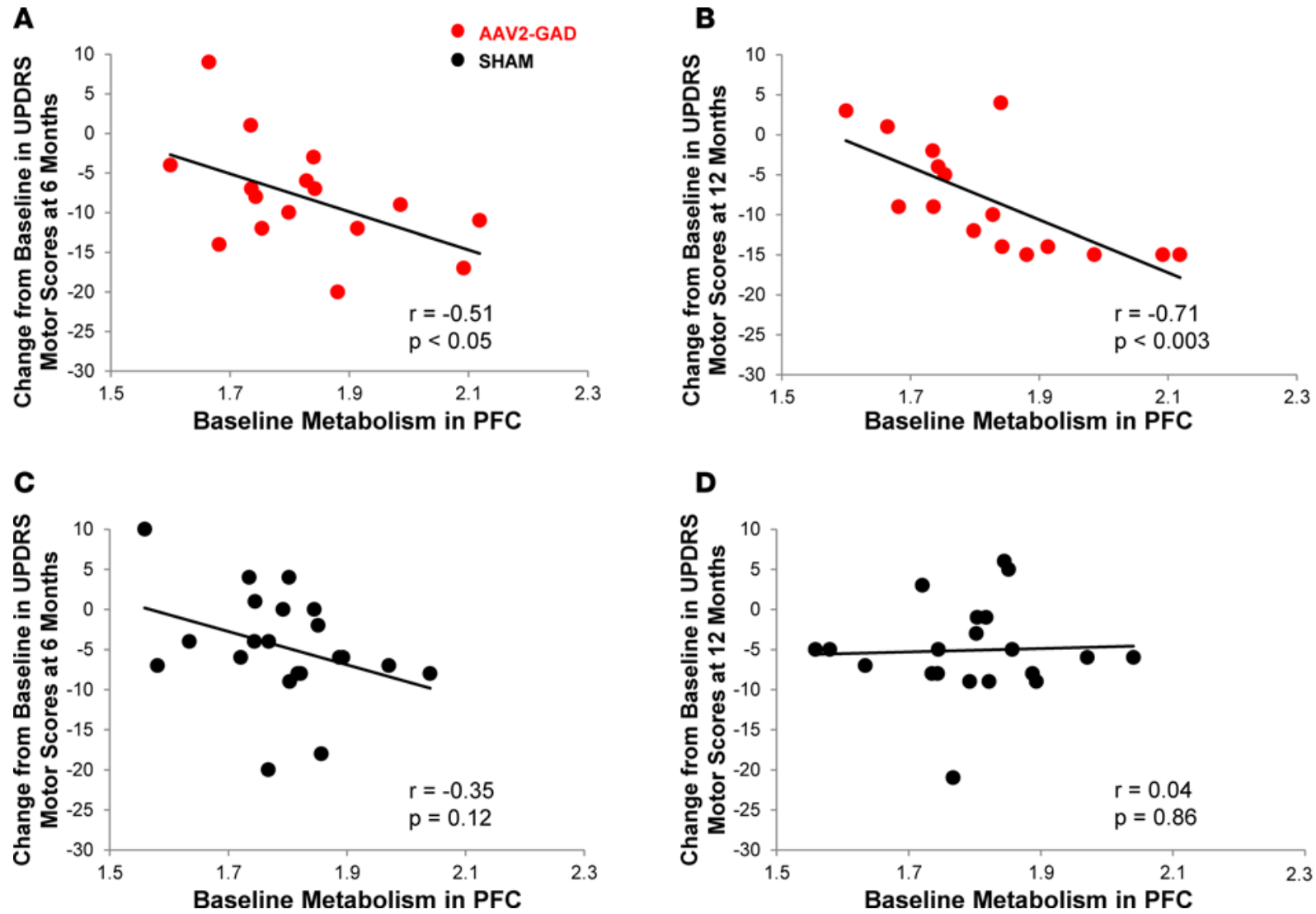

Figure 3. Correlation between baseline metabolism in the prefrontal cortex (PFC) and clinical response. In the AAV2-CAD group ( $n=16$ ), an inverse correlation was evident between the baseline metabolism in the PFC and the changes in Unified Parkinson's Disease Rating Scale (UPDRS) motor scores at 6 months (A: $r=-0.51, P<0.05$; Pearson's correlation) and 12 months (B: $r=-0.71, P<0.003$; Pearson's correlation) from baseline. No correlation was seen in the sham group $(n=21)$ at either 6 months $(C: r=-0.35, P=0.12$; Pearson's correlation) or 12 months ( $\mathbf{D}: r=-0.04, P=0.86$; Pearson's correlation).

anterior cingulate circuit (19). Medial prefrontal cortex also projects to the STN; perhaps by a similar mechanism, STN AAV2-GAD treatment also results in decreased resting-state metabolism in prefrontal cortex. Interestingly, though levodopa improves motor function in $\mathrm{PD}$, it also suppresses prefrontal cortex activation responses (20). The correlation between baseline prefrontal cortex metabolism and the response to the AAV2-GAD therapy (the higher the baseline metabolism, the better the response) may reflect an enhanced benefit for patients with less cortical involvement, rather than indicating that changes in prefrontal cortex activity underlie the motor benefit seen in this trial. In other words, though baseline prefrontal cortex metabolism predicted UPDRS response, the change in prefrontal cortex metabolism did not correlate with improvement (data not shown) $(16,21)$. These data also suggest the possibility that, in addition to assuring an accurate diagnosis of idiopathic PD, screening FDG PET imaging could also be utilized to select for subjects that are more likely to respond to this therapy. We note that subjects classified as responders at 12 months tended to have higher baseline prefrontal cortex metabolism (Figure 3B). Thus, an inclusion criterion that sets a minimum standard for baseline prefrontal cortex metabolism could be used to increase statistical power and reduce the sample size needed for a future clinical trial. Nonetheless, this will need to be confirmed prospectively in a larger trial powered for efficacy. Utilizing prefrontal metabolism as a screening criteria could limit the generalizability of a future clinical trial and, based only on our preliminary exploratory analysis, should be undertaken with caution.

In summary, the 12-month open-label follow-up data from our 6-month double-blind sham surgery-controlled clinical trial of STN AAV2-GAD in patients with advanced PD (4) demonstrate continued motoric benefits of the therapy. In addition, the imaging results suggest that FDG PET may have utility as a screening procedure for clinical trials of PD therapeutics (especially small-scale phase I and II studies) and may provide insights into the mechanisms underlying novel therapies. 


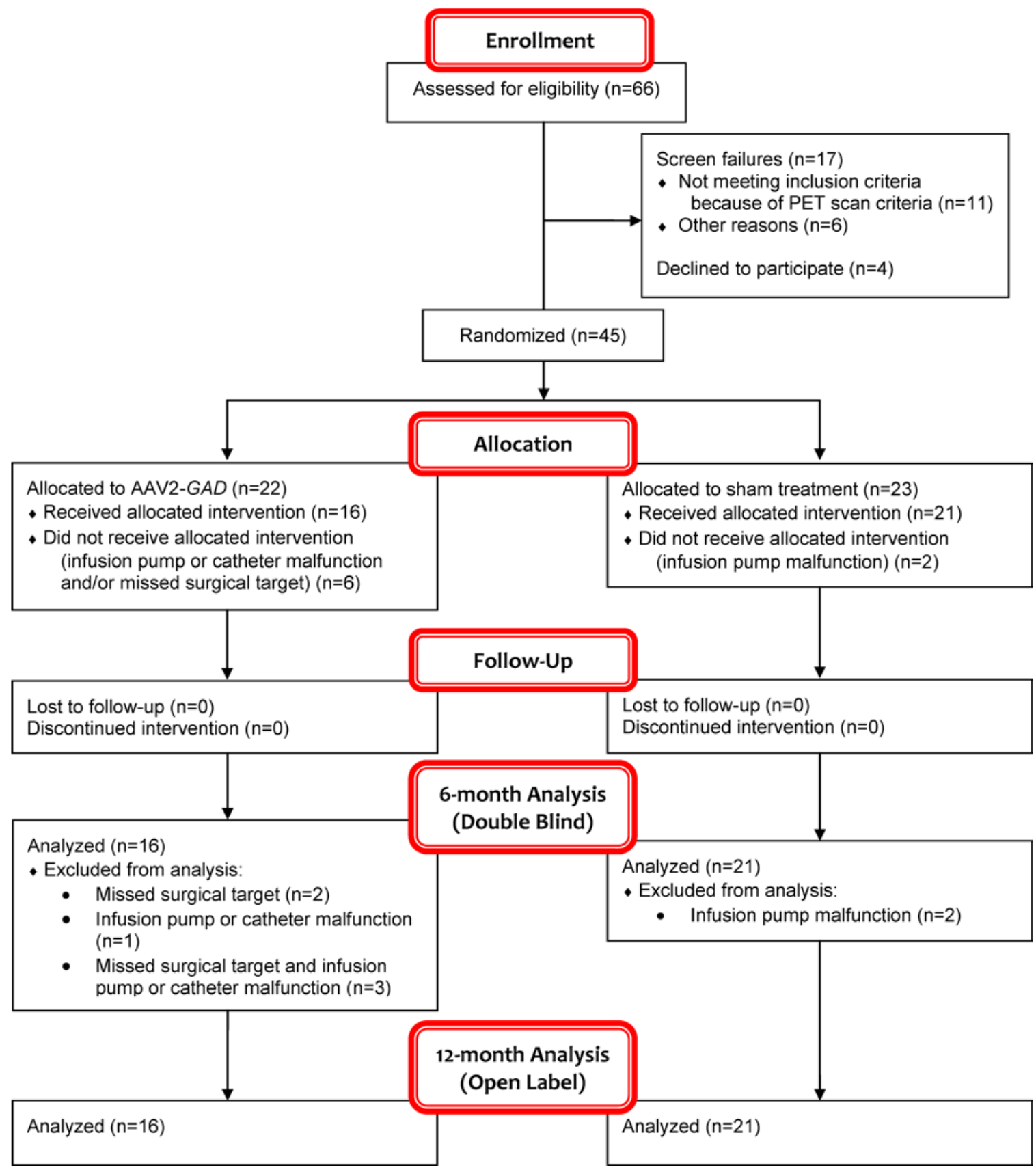

Figure 4. Trial profile.

\section{Methods}

Subjects. As described in the report on the 6-month data, 66 subjects were screened for the trial at 7 sites, and 45 were randomized (4). 22 were assigned to AAV2-GAD and 23 to sham surgery (Figure 4). Ten subjects were excluded prior to imaging, and eleven subjects were excluded based upon analysis of their initial FDG PET scan (see below) according to previously published criteria of image-based differential diagnosis (22). An additional 8 subjects were excluded from the data analysis prior to unblinding due to failure of drug delivery (pump failure, inaccurate targeting of STN), based upon predetermined criteria. The characteristics of the remaining 37 subjects have been described in detail previously (4). There was no significant difference in levodopa equivalent dose at baseline and 6 months for either group or between groups (sham: baseline, 1,125.93 $\pm 493.8 \mathrm{mg} ; 6$ month, 1,095.23 $\pm 499.9 \mathrm{mg}$; AAV2-GAD: baseline, 1,149.02 $\pm 536.3 \mathrm{mg} ; 6$ month, 1,135 $\pm 560.9 \mathrm{mg}$ ). Investigators were instructed to try to avoid 


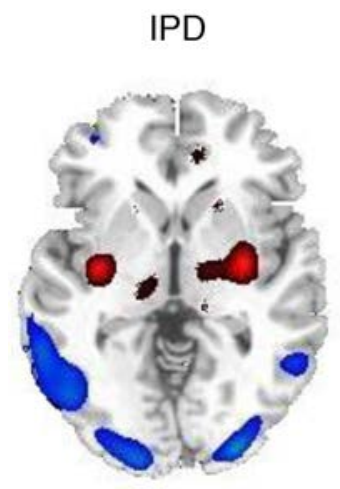

$z=-1$

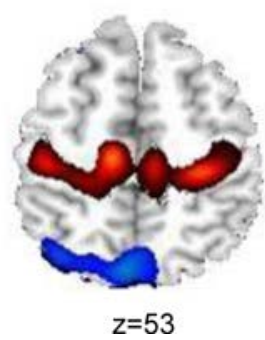

MSA
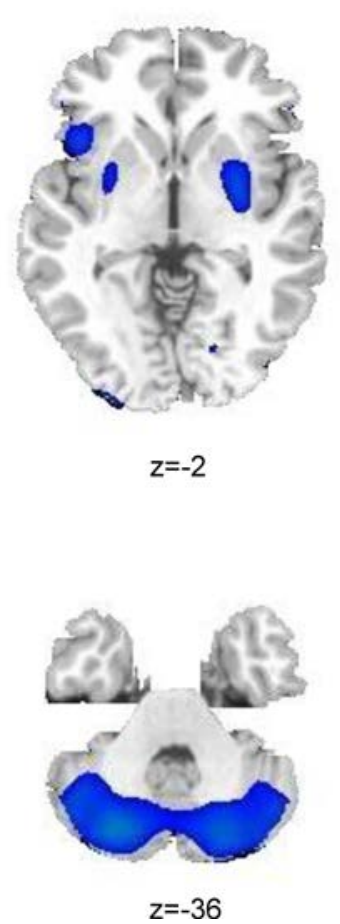

Figure 5. Display of abnormal glucose metabolism $(P<0.001$, uncorrected, by SPM $t$ test) in two representative patients using the statistical parametric mapping single-case analysis (26). (Increased/decreased metabolism is indicated by red/blue.) Based on the automated image-based diagnosis for individual subjects (22), one patient was classified as having idiopathic Parkinson's disease (IPD) with a likelihood of $99 \%$, consistent with this subject's clinical diagnosis, and was enrolled into the study. The other subject was classified as having multiple system atrophy (MSA) with a likelihood of $99 \%$, despite a clinical diagnosis of IPD, and thus was excluded from the study.

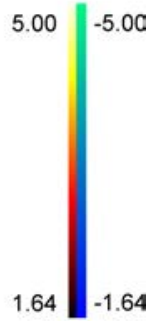

changing PD medications over the course of the year, but doses of antiparkinsonian medications were not formally tracked in the open-label phase of the trial.

In addition, imaging data from 22 healthy subjects (age $60.7 \pm 10.0$ years), age matched to the trial subjects, were selected from a historical data set and served as an additional control group for the imaging analysis.

Study design. This was a multicenter, randomized, double-blind, sham surgery-controlled clinical trial. Subjects were assessed clinically at baseline and after 1 , 3,6 , and 12 months. The primary outcome measure was the difference from baseline in the OFF-state total motor UPDRS score (part 3) over 12 months between the sham

and AAV2-GAD groups. Key secondary outcome measures included ON-state motor UPDRS score, UPDRS Activities of Daily Living score (part 2), Brief Parkinsonism Rating Scale, UPDRS complications of therapy scale (part 4), such as daily duration of LID and of OFF time, and an extensive neuropsychological test battery. FDG PET was done at baseline and repeated at 6 and 12 months for all subjects. Details regarding other assessments have been previously reported (4).

FDG PET. FDG PET scanning was done at 5 participating sites. PET studies were performed in 3D mode according to protocols described in detail elsewhere (23). Subjects underwent scanning on the PET scanner available at each facility, except for those enrolled at the Ohio State University College of Medicine and the University of Colorado School of Medicine, who underwent scanning at the Feinstein Institute for Medical Research. All subjects fasted overnight prior to PET scanning and were positioned in a stereoadapter. To minimize repositioning errors, the settings employed in the baseline scan were also used for the second (after 6 months) and third scans (after 12 months). A transmission scan (10 minutes) was acquired for attenuation correction in emission scans. A CT transmission scan ( $<1$-minute duration) was performed just before or after the emission scan at those sites utilizing a PET/CT machine. All images had an effective image resolution of $6 \mathrm{~mm}$ transversely and $8 \mathrm{~mm}$ axially. FDG PET images were acquired for 20 minutes (two 10-minute frames), beginning 35 minutes after the injection of $0.071 \mathrm{mCi} / \mathrm{kg}(\sim 5 \mathrm{mCi}) \mathrm{FDG}$ in a resting state with eyes open and with minimal auditory stimulation.

FDG PET scan data were transferred in Dicom format via a secure server to the central imaging coordination center at the Feinstein Institute for Medical Research. All imaging analyses, including network computations, were performed only at the Feinstein Institute for Medical Research using in-house software (available at http://feinsteinneuroscience.org/). This program is implemented in MATLAB (The Mathworks Inc.) on a PC-based workstation. Scans were also analyzed utilizing statistical parametric mapping (SPM5, Wellcome Trust Centre for Neuroimaging, Institute of Neurology, London, United Kingdom) for additional image preprocessing and statistical analysis. All imaging analyses were performed blind to group designation (i.e., AAV2-GAD versus sham).

Initial imaging analysis of baseline scans was undertaken during the screening process prior to randomization to confirm the diagnosis of idiopathic PD. These baseline scans were assessed in real time using an automated differential diagnosis algorithm based upon previously identified disease-specific brain networks for idiopathic PD (PD-related pattern), MSA, and progressive supranuclear palsy (22). For entry into the study, the estimated probability of idiopathic $\mathrm{PD}$ for each subject needed to be $>81 \%$, an image-based diag- 
nostic criterion established in a prior study (22). Of the 56 subjects screened with imaging, 45 met this criterion and were enrolled into the study (4). Four subjects, whose probabilities of idiopathic PD were found to be $<21 \%$ (or, alternatively, probabilities of atypical PD of $>79 \%$ ), were classified as MSA by the algorithm and were thus excluded from the study. The images of one representative idiopathic PD subject and one subject with MSA are shown in Figure 5. Moreover, 7 additional subjects whose probabilities of idiopathic PD were between $21 \%$ and $80 \%$ were classified as indeterminate cases and were excluded as well. Imaging results of the baseline scans were returned to the site investigators within 24 hours.

FDG PET scans were analyzed by comparing changes across 3 time points (baseline, 6 months, and 12 months) between the AAV2-GAD and sham groups, utilizing an SPM5 $2 \times 3$ RMANOVA model. In each of the significant areas identified, regional metabolic activity was measured and compared between groups over time.

Statistics. Two-tailed Student's $t$ tests were used to examine the differences in baseline values of each clinical outcome (e.g., the OFF-state motor UPDRS score) or imaging measure (regional metabolism) between the AAV2-GAD and sham groups. Two-tailed Student's $t$ tests were also used to compare the differences in regional metabolism at baseline between the AAV2-GAD or sham group and the healthy control group. Pearson's correlation coefficients were calculated to examine the relationship between baseline metabolism in each region and the changes in UPDRS motor scores at 6 and 12 months from baseline. In both treatment groups, a secondary post-hoc analysis was also performed for 12-month responder data. This analysis was done on the raw (i.e., not adjusted for baseline scores) UPDRS motor score data. We defined a clinically meaningful response as a 29.0-point improvement in UPDRS motor score, which corresponds to the mean improvement of $25 \%$ in the initial AAV2-GAD study (24) and to a moderate-to-large clinically important difference reported in an analysis of treatments for PD (25). Pearson's $\chi^{2}$ test was used to examine differences in the proportion of responders based on changes in UPDRS or diary ON/OFF times between the AAV2-GAD and sham groups.

To assess longitudinal changes of the primary outcome measure, the OFF-state motor UPDRS score was analyzed by 2-way RMANOVA over all 5 time points (baseline and 1, 3, 6, and 12 months), with treatment group as the between-subjects measure and time point as the within-subject repeated measure. Post-hoc Bonferroni tests were used to assess the changes at follow-up time points with respect to baseline. Secondary efficacy measures were analyzed using the same method. Changes in regional metabolic activity over the 12-month period were also analyzed using 2-way RMANOVA.

For display of data in the figures, if no significant group difference was found at baseline, we subtracted each subject's baseline values from the values of that subject at all time points, such that only the changes of each measure from baseline were displayed at follow-up time points.

The statistical analysis was performed with SAS 9.3 (SAS Institute). Results were considered significant at $P<0.05$.

Study approval. Study protocols and consent forms were approved by the institutional review boards of Stanford University, University of Colorado, Massachusetts General Hospital, Henry Ford Health Systems - Franklin Pointe Medical (Southfield, Michigan), University of Rochester, Wake Forest University Health Science Center, the Ohio State University, and the Feinstein Institute for Medical Research at Northwell Health, as described previously (4). Written consent was obtained from every patient after detailed explanation of the procedures.

\section{Author contributions}

PAL, MJD, MGK, and AF conceived of the research project. PAL, CVS, MJD, MGK, and AF organized the research project. PAL, ARR, MAL, SGO, AWF, ENE, SKK, AS, MSS, SBT, JMS, KLP, JMH, RMK, IHR, CVS, MJD, MGK, and AF executed the research project. MJD, MGK, and AF designed the statistical analysis. CCT, MJD, MGK, and AF executed the statistical analysis. Review and critique of the statistical analysis was performed by MN, CCT, MJD, MGK, DE, and AF. MN and AF wrote the first draft of the manuscript. Review and critique of the manuscript was performed by all authors.

\section{Acknowledgments}

This work was supported by Neurologix Inc.

Address correspondence to: Andrew Feigin, Center for Neurosciences, The Feinstein Institute for Medical Research, 350 Community Drive, Manhasset, New York 11030, USA. Phone: 516.562.2498; E-mail: afeigin@northwell.edu. 
1. Bevan MD, Atherton JF, Baufreton J. Cellular principles underlying normal and pathological activity in the subthalamic nucleus. Curr Opin Neurobiol. 2006;16(6):621-628.

2. Nutt JG. Motor fluctuations and dyskinesia in Parkinson's disease. Parkinsonism Relat Disord. 2001;8(2):101-108

3. Fenoy AJ, Simpson RK. Risks of common complications in deep brain stimulation surgery: management and avoidance. $J$ Neurosurg. 2014;120(1):132-139.

4. LeWitt PA, et al. AAV2-GAD gene therapy for advanced Parkinson's disease: a double-blind, sham-surgery controlled, randomised trial. Lancet Neurol. 2011;10(4):309-319.

5. Mittermeyer G, et al. Long-term evaluation of a phase 1 study of AADC gene therapy for Parkinson's disease. Hum Gene Ther. 2012;23(4):377-381.

6. Palfi S, et al. Long-term safety and tolerability of ProSavin, a lentiviral vector-based gene therapy for Parkinson's disease: a dose escalation, open-label, phase 1/2 trial. Lancet. 2014;383(9923):1138-1146.

7. Peng S, et al. Characterization of disease-related covariance topographies with SSMPCA toolbox: effects of spatial normalization and PET scanners. Hum Brain Mapp. 2014;35(5):1801-1814.

8. Deuschl G, et al. A randomized trial of deep-brain stimulation for Parkinson's disease. N Engl J Med. 2006;355(9):896-908

9. Ko JH, et al. Network modulation following sham surgery in Parkinson's disease. J Clin Invest. 2014;124(8):3656-3666.

10. Schwingenschuh $P$, et al. Distinguishing SWEDDs patients with asymmetric resting tremor from Parkinson's disease: a clinical and electrophysiological study. Mov Disord. 2010;25(5):560-569.

11. Whone AL, et al. Slower progression of Parkinson's disease with ropinirole versus levodopa: The REAL-PET study. Ann Neurol. 2003;54(1):93-101.

12. Marek KI, Seibyl J, Parkinson Study Group. $\beta$-CIT scans without evidence of dopaminergic deficit (SWEDD) in the ELLDOPA-CIT and CALM-CIT study: long-term imaging assessment. Neurology. 2003;60(Supp1. 1):A293.

13. Feigin A, et al. Modulation of metabolic brain networks after subthalamic gene therapy for Parkinson's disease. Proc Natl Acad Sci USA. 2007;104(49):19559-19564.

14. Feigin A, et al. Metabolic correlates of levodopa response in Parkinson's disease. Neurology. 2001;57(11):2083-2088

15. Asanuma K, et al. Network modulation in the treatment of Parkinson's disease. Brain. 2006;129(Pt 10):2667-2678.

16. Schroeder U, et al. Subthalamic nucleus stimulation affects striato-anterior cingulate cortex circuit in a response conflict task: a PET study. Brain. 2002;125(Pt 9):1995-2004.

17. Limousin P, Greene J, Pollak P, Rothwell J, Benabid AL, Frackowiak R. Changes in cerebral activity pattern due to subthalamic nucleus or internal pallidum stimulation in Parkinson's disease. Ann Neurol. 1997;42(3):283-291.

18. Ceballos-Baumann AO, et al. A positron emission tomographic study of subthalamic nucleus stimulation in Parkinson disease: enhanced movement-related activity of motor-association cortex and decreased motor cortex resting activity. Arch Neurol. 1999;56(8):997-1003.

19. Alexander GE, Crutcher MD, DeLong MR. Basal ganglia-thalamocortical circuits: parallel substrates for motor, oculomotor, "prefrontal" and "limbic" functions. Prog Brain Res. 1990;85:119-146.

20. Cools R, Stefanova E, Barker RA, Robbins TW, Owen AM. Dopaminergic modulation of high-level cognition in Parkinson's disease: the role of the prefrontal cortex revealed by PET. Brain. 2002;125(Pt 3):584-594

21. Chudasama Y, Baunez C, Robbins TW. Functional disconnection of the medial prefrontal cortex and subthalamic nucleus in attentional performance: evidence for corticosubthalamic interaction. J Neurosci. 2003;23(13):5477-5485.

22. Tang CC, et al. Differential diagnosis of parkinsonism: a metabolic imaging study using pattern analysis. Lancet Neurol. 2010;9(2):149-158.

23. Ma Y, Tang C, Spetsieris PG, Dhawan V, Eidelberg D. Abnormal metabolic network activity in Parkinson's disease: test-retest reproducibility. J Cereb Blood Flow Metab. 2007;27(3):597-605.

24. Kaplitt MG, et al. Safety and tolerability of gene therapy with an adeno-associated virus (AAV) borne GAD gene for Parkinson's disease: an open label, phase I trial. Lancet. 2007;369(9579):2097-2105.

25. Shulman LM, Gruber-Baldini AL, Anderson KE, Fishman PS, Reich SG, Weiner WJ. The clinically important difference on the unified Parkinson's disease rating scale. Arch Neurol. 2010;67(1):64-70.

26. Eckert T, et al. FDG PET in the differential diagnosis of parkinsonian disorders. Neuroimage. 2005;26(3):912-921 